\title{
Notificações de erros de medicação em um hospital geral de urgência e emergência
}

\author{
Notifications of medication errors in a general hospital of urgency and emergency \\ Notificaciones de errores de medicación en un hospital general de urgencia y emergência
}

Recebido: 26/05/2021 | Revisado: 04/06/2021 | Aceito: 09/06/2021 | Publicado: 23/06/2021

Diana Silva Lopes

ORCID: https://orcid.org/0000-0002-8135-6652 Universidade Estadual do Sudoeste da Bahia, Brasil E-mail: dianasilvalopes@hotmail.com

Ana Mercia Silva Mascarenhas

ORCID: https://orcid.org/0000-0001-6116-5691 Universidade Estadual do Sudoeste da Bahia, Brasil E-mail: anamercia.sm@gmail.com

Nara Jacqueline Souza dos Santos

ORCID: https://orcid.org/0000-0003-0874-5771

Universidade Estadual do Sudoeste da Bahia, Brasil E-mail: nara.jacqueline@gmail.com

Tamiles Daiane Borges Santana ORCID: https://orcid.org/0000-0003-4466-5031

Universidade Estadual do Sudoeste da Bahia, Brasil E-mail: tamilesdbs@ hotmail.com

Tuany Santos Souza

ORCID: https://orcid.org/0000-0003-0165-4201 Universidade Estadual do Sudoeste da Bahia, Brasil E-mail: tuanysouza.s@uesb.edu.br

Julita Maria Pereira Borges

ORCID: https://orcid.org/0000-0003-3969-8146 Universidade Estadual do Sudoeste da Bahia, Brasil E-mail: jmpborges@uesb.edu.br Gisele da Silveira Lemos

ORCID: https://orcid.org/0000-0001-8987-0245 Universidade Estadual do Sudoeste da Bahia, Brasil E-mail: giselesilveiralemos@gmail.com

\begin{abstract}
Resumo
Os eventos adversos relacionados a medicamentos podem resultar em repercussões socioconômicas e agravos significativos aos pacientes. Sendo assim, o estudo teve como objetivo a avaliação das notificações de erros de medicação feitas ao Núcleo de Segurança do Paciente de um hospital de urgência e emergência no interior da Bahia. Estudo transversal, descritivo-analítico, realizado em um hospital público de urgência e emergência. Os dados foram coletados a partir das notificações de eventos adversos feitas durante três meses de coleta. O tipo e a classificação do erro foram as variáveis consideradas. O teste de Kolmogorov-Smirnov foi utilizado para avaliação da normalidade das variáveis contínuas, considerando p>0,05 distribuição normal e para comparar as proporções das variáveis categóricas foi utilizado o teste Quiquadrado de Pearson e exato de Fisher. Identificou-se 151 notificações de erros de medicação, classificados por tipo: prescrição $(51,7 \%)$, administração $(45,7 \%)$ e dispensação $(2,6 \%)$. Estes receberam 12 classificações, sendo as mais frequentes: dose incorreta $(42,4 \%)$, omissão do medicamento $(26,5 \%)$ e horário de administração (7,9\%). A maior ocorrência de notificação de erros foi na Unidade de Terapia Intensiva (46,4\%). As classes medicamentas mais envolvidas com os erros foram as do trato alimentar e metabolismo, sistema cardiovascular e anti-infecciosos de uso sistêmico. Dentre os profissionais notificadores os farmacêuticos foram os que mais realizaram notificações. Diante das notificações identificadas e considerando que erros de medicação são eventos evitáveis, sugere-se que esforços sejam dedicados na busca de mais informações para uma melhor avaliação e conhecimento das consequências e possíveis danos relacionados aos erros de medicação.
\end{abstract}

Palavras-chave: Erros de medicação; Segurança do paciente; Notificação.

\begin{abstract}
Drug-related adverse events can result in socioeconomic repercussions and harm to patients. Thus, the study aimed to evaluate the notifications of medication errors made to the Patient Safety Center of an emergency and emergency hospital in the interior of Bahia. Cross-sectional, descriptive-analytical study carried out in a public emergency and emergency hospital. Data were collected from adverse event notifications made during three months of collection. The type and classification of the error were as determined. The Kolmogorov-Smirnov test was used to assess the normality of the continuous variables, considering $p>0.05$ as normal distribution, and to compare the proportions of the categorical variables, Pearson's chi-square test and Fisher's exact test were used. 151 notifications of medication
\end{abstract}


errors were identified, classified by type: prescription (51.7\%), administration (45.7\%) and dispensing (2.6\%). These classifications, 12 classifications, being the most frequent: incorrect dose (42.4\%), medication omission $(26.5 \%)$ and administration time (7.9\%). The highest occurrence of errors was in the Intensive Care Unit (46.4\%). The drug classes most involved with errors were those related to the alimentary tract and metabolism, cardiovascular system and antiinfectives for systemic use. Among the notifying professionals, pharmacists were the ones who performed the most notifications. In view of the identified notifications and considering that medication errors are preventable events, efforts are necessarily made in the search for more information for a better assessment and knowledge of the consequences and possible damages related to medication errors.

Keywords: Medication errors; Patient safety; Notification.

\section{Resumen}

Los eventos adversos relacionados con medicamentos pueden tener repercusiones socioeconómicas y daños a los pacientes. Así, el estudio tuvo como objetivo evaluar las notificaciones de errores de medicación realizadas al Centro de Seguridad del Paciente de un hospital de urgencias y emergencias del interior de Bahía. Estudio descriptivoanalítico de corte transversal realizado en un hospital público de urgencias y emergencias. Se recopilaron datos de las notificaciones de eventos adversos realizadas durante tres meses. Se determinó el tipo y la clasificación del error. Se utilizó la prueba de Kolmogorov-Smirnov para evaluar la normalidad de las variables continuas, considerando p>0,05 como distribución normal, y para comparar las proporciones de las variables categóricas, se utilizó la prueba de chicuadrado de Pearson y la prueba exacta de Fisher. Se identificaron 151 notificaciones de errores de medicación, clasificadas por tipo: prescripción $(51,7 \%)$, administración $(45,7 \%)$ y dispensación $(2,6 \%)$. Destas 12 clasificaciones, las más frecuentes: dosis incorrecta $(42,4 \%)$, omisión de medicación $(26,5 \%)$ y tiempo de administración $(7,9 \%)$. La mayor ocurrencia de errores fue en la Unidad de Cuidados Intensivos $(46,4 \%)$. Las clases de fármacos más implicadas en errores fueron relacionadas con el tracto digestivo y el metabolismo, sistema cardiovascular y antiinfecciosos de uso sistémico. Entre los profesionales notificantes, los farmacéuticos realizaron más notificaciones. Ante las notificaciones identificadas y considerando que los errores de medicación son eventos prevenibles, necesariamente se hace un esfuerzo en la búsqueda de mayor información para una mejor valoración y conocimiento de las consecuencias y posibles daños relacionados con los errores de medicación.

Palabras clave: Errores de Medicación; Seguridad del Paciente; Notificación.

\section{Introdução}

Os eventos adversos (EA) relacionados a medicamentos podem resultar em relevantes repercussões sociais, econômicas e agravos significativos à saúde dos pacientes. Os erros de medicação se destacam dentre esses eventos, por serem evitáveis, frequentes e terem consequências impactante (Santi et al., 2014), uma vez que a ocorrência de incidentes relacionados aos cuidados de saúde pode representar uma elevação nas taxas de morbimortalidade nos serviços de saúde (Santi et al., 2014).

Define-se como erro de medicação qualquer evento evitável que, de fato ou potencialmente, pode levar ao uso inadequado do medicamento, causando dano ou não ao paciente, e não importando se o medicamento se encontra sob o controle de profissionais de saúde, dos pacientes ou dos consumidores (NCCMEP, 2015). O erro pode estar associado à prática profissional, produtos e sistemas, incluindo prescrição, rotulagem, nomenclatura, preparação, dispensação, distribuição, administração, educação, monitoramento e utilização (NCCMEP, 2015).

Nos Estados Unidos, os erros causam pelo menos uma morte por dia e aproximadamente 1,3 milhões de pessoas são atingidas por danos anualmente (OMS, 2017). Esses números se assemelham em países em desenvolvimento como o Brasil. Os custos associados aos erros de medicação chegam a US\$ 42 bilhões, o que corresponde a quase $1 \%$ do total de despesas em saúde no mundo5. No entanto, esses valores podem ser ainda maiores (Watanabe, McInnis, \& Hirsch, 2018) que estimaram em US\$ 528,4 bilhões os custos com morbimortalidade relacionada a prescrições de medicamentos de terapia não otimizadas.

No cenário brasileiro alguns estudos de forma regional exemplificam a ocorrência de erros de medicação, como demonstrado em um hospital universitário do Ceará, onde a prevalência de erros de prescrição clinicamente significativos foi de 292,5 erros por 1000 itens prescritos (Néri et al., 2011). No estudo de Silva et al. (J. S. D. S. Silva et al., 2017) os erros de administração de maior ocorrência foram os de dose administrada incorreta, ausência da identificação do paciente no leito, técnica incorreta de administração e horário incorreto. Já Mendes et al. (Mendes, 2018) identificaram erros no preparo de 
medicamentos, como a não higienização das mãos (70,29\%) e não utilização de técnica asséptica (80,85\%). Meira et al. (Meira et al., 2020) encontrou erros de prescrição como ausência da informação sobre o diluente $(97,1 \%)$, velocidade de infusão $(99,4 \%)$, dose incorreta $(5,8 \%)$ e abreviaturas proibidas $(1,1 \%)$. E para erros de administração observou-se os tipos de erros de falta de identificação do paciente pelo profissional $(94,3 \%)$, falta de identificação do paciente no leito $(12,1 \%)$, horário de administração $(6,3 \%)$ e técnica incorreta de preparo e administração do medicamento $(1,1 \%)$.

Cabe destacar que em 2017, a Organização Mundial de Saúde (OMS, 2017) estabeleceu como prioridade para a segurança dos pacientes, a adoção de medidas que minimizem os erros relacionados a medicamentos. Definiu-se, mundialmente, uma meta de redução em 50\% dos erros de medicação para os próximos cinco anos (OMS, 2017). E, com o Brasil ocupando a sexta posição entre os maiores mercados consumidores de medicamentos no cenário mundial (CFF, 2018), faz-se necessário mais estudos sobre a magnitude do problema dos erros de medicação.

Diante do contexto, há interesse em avaliar os erros de medicação com a pretensão de conhecer os dados e definir medidas de prevenção, visando aumentar a segurança do paciente para tais eventos. Sendo assim, a realização do presente estudo teve como objetivo a avaliação das notificações de erros de medicação feitas ao Núcleo de Segurança do Paciente de um hospital de urgência e emergência no interior da Bahia.

\section{Metodologia}

\subsection{Tipo e local de estudo}

O estudo caracteriza-se como observacional, transversal, descritivo-analítico, tendo como local da pesquisa um hospital público, no interior do estado da Bahia (Pereira et al., 2018). O referido hospital é geral, referência estadual e responsável pelos atendimentos secundários e terciários de especialidades e procedimentos de média e alta complexidade. Dessa forma, está definido como Hospital Estratégico da Rede de Atenção às Urgências, por ser referência regional para 27 municípios da microrregião de saúde ao qual está inserido (SESAB, 2018).

Este hospital possui um total de 276 leitos, sendo 29 destinados a unidades de terapia intensiva (UTI). Os demais leitos compõem as clínicas médica, cirúrgica, ortopédica, pediatria, psiquiatria e emergência adulta e pediátrica. Possui também um Núcleo de Segurança do Paciente, criado em 2015 e composto por uma equipe multiprofissional de saúde com enfermeiros, médicos, farmacêuticos, fisioterapeuta e nutricionista. A presente instituição também conta com o apoio de uma Residência Multiprofissional com atuação em Farmácia Clínica.

\subsection{Instrumento e coleta de dados}

$\mathrm{O}$ instrumento de coleta utilizado foi um formulário baseado nas informações das fichas de notificação de eventos adversos relacionados a medicamentos em uso no hospital, confeccionada pelo Núcleo de Segurança do Paciente (NSP).

Os dados coletados foram referentes aos meses de maio, junho e julho de 2019, com análise de todas as fichas de notificação de eventos adversos recebidas pelo NSP do hospital, independente de terem sido notificadas ao sistema de Notificações em Vigilância Sanitária (Notivisa) da Agência Nacional de Vigilância Sanitária (Anvisa).

As notificações foram apontadas por 13 setores do hospital. Sendo estes categorizadas em: UTI (UTI 1, UTI 2 e UTI 3), emergência (sala verde, sala amarela, sala de especialidades e sala vermelha) e clínicas (médica, neurológica, cirúrgica, vascular, pediatria e internamento não especificado).

\subsection{Variáveis}

A variável dependente analisada foi tipo de erro e erro de medicação, Figura 1, apontada na notificação de eventos adversos relacionados a medicamentos e as variáveis independentes foram: sexo, idade, unidade de internação, medicamento, 
classe terapêutica e profissional notificador.

Os medicamentos identificados nas notificações foram classificados conforme a classificação Anatômica Terapêutico Química (ATC, do inglês, Anatomical Therapeutic Chemical) nível 1, da Organização Mundial de Saúde (OMS), que representa o grupo anatômico principal (OMS, 2019).

Figura 1 - Fluxograma de classificação, tipo e causas de erros de medicamentos utilizados no estudo.

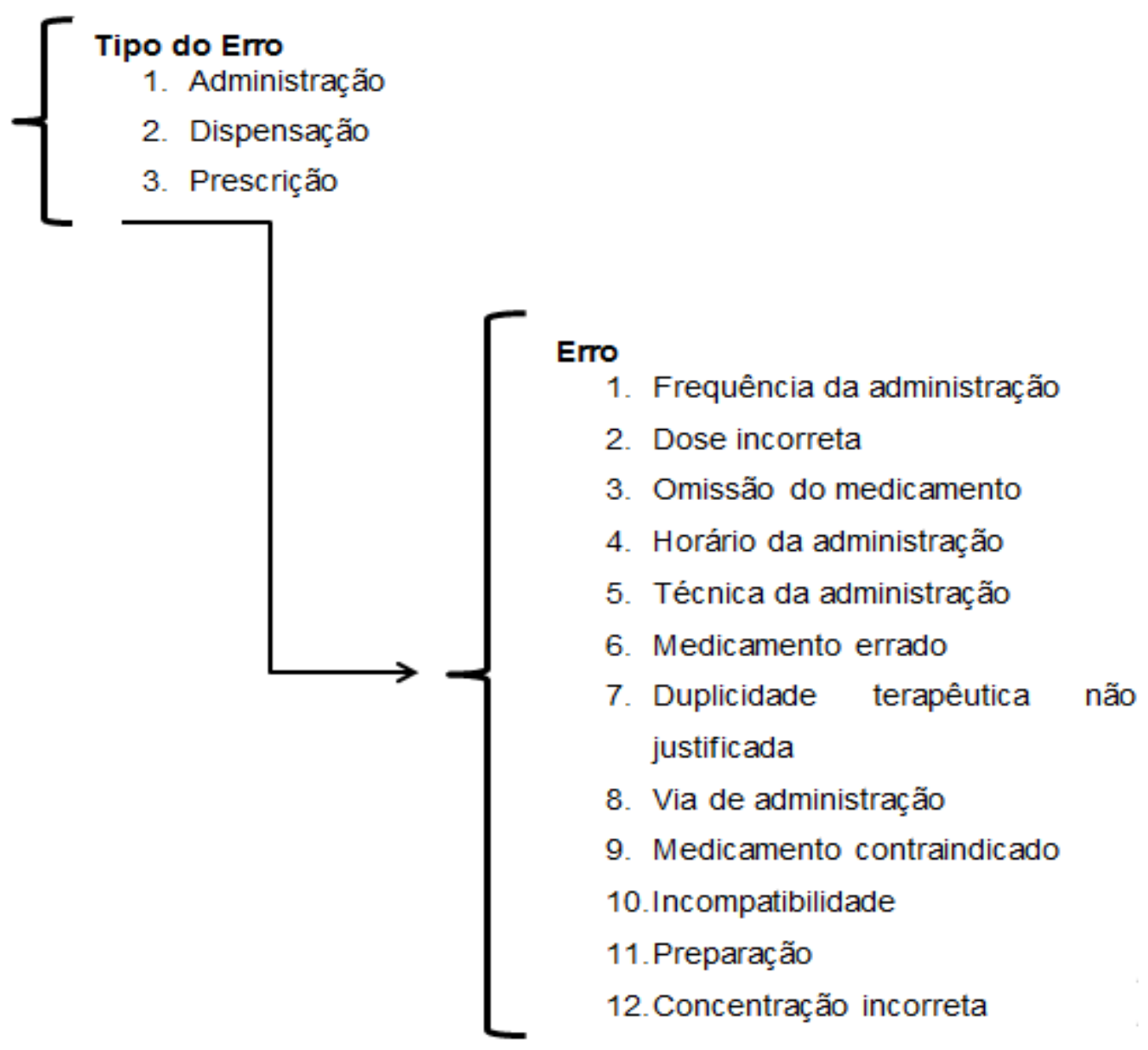

Fonte: Elaboração dos autores.

Os tipos de erros foram definidos conforme Anacleto (Anacleto et al., 2010), em:

Erros de prescrição: erros de decisão ou de redação, não intencionais, que podem reduzir a probabilidade do tratamento ser efetivo ou aumentar o risco de lesão no paciente.

Erros de dispensação: são os erros que estão mais relacionados com as atividades da farmácia hospitalar e englobam os erros de conteúdo (medicamento errado; concentração errada; forma farmacêutica errada; medicamento com desvio de qualidade; dispensação de medicamento prescrito sem horário, quantidade, concentração ou forma farmacêutica), rotulagem (podem gerar dúvidas no momento da dispensação e/ou administração) e documentação (ausência ou registro incorreto da dispensação de medicamentos controlados, falta de data na prescrição, falta de assinatura do prescritor ou do dispensador).

Erros de administração: qualquer desvio no preparo e administração de medicamentos mediante prescrição médica, não observância dos protocolos e procedimentos operacionais padrão do hospital ou das instruções técnicas do fabricante do produto.

Cada erro foi especificado pela descrição do erro notificado. O padrão de identificação dos erros de medicação seguiu 
a denominação definida por Anacleto et al. (Anacleto et al., 2010). As informações referentes a avaliação da dose, frequência de administração da dose, via de administração, contraindicação e técnica de administração, foram avaliadas considerando as informações contidas na bula do medicamento aprovado pela Anvisa, disponível na bula do Profissional do bulário eletrônico dessa Agência (Brasil, 2021).

Sendo assim foi considerado:

- frequência da administração: intervalo maior ou menor do que o recomendado ou prescrito (para erro de administração).

- dose incorreta: dose maior ou menor do que o recomendado ou prescrito de acordo com sua condição clínica (alterações renais, hepáticas, peso).

- omissão do medicamento: não administração do medicamento prescrito, falta do medicamento na farmácia, ou patologia não tratada com terapia medicamentosa.

- $\quad$ horário da administração: horário inadequado para administração, ou atraso no horário aprazado (30 minutos antes ou 30 minutos depois deste horário).

- t técnica da administração: administração do medicamento de forma não indicada.

- medicamento errado: troca do medicamento, troca de paciente, medicamento não indicado.

- duplicidade terapêutica não justificada: medicamentos com mesma indicação sem adição de benefício terapêutico, mesmo medicamento e posologia prescritos duas vezes na mesma prescrição.

- $\quad$ via de administração: via de administração incorreta, contraindicada.

- medicamento contraindicado: medicamento não indicado para $o$ paciente, por interação ou incompatibilidade.

- $\quad$ incompatibilidade: medicamentos incompatíveis para administração concomitante.

- $\quad$ preparação: reconstituição, diluição, trituração ou partição inadequada.

- $\quad$ concentração incorreta: dose incorreta para o volume ou dose inadequada para indicação.

\subsection{Análise de dados}

As análises descritivas dos dados foram realizadas através da distribuição de frequências absoluta e relativa das variáveis categóricas, apresentadas no formato de tabelas, e através de medidas de tendência central (média e mediana) e de dispersão (desvio-padrão e intervalo interquartílico) das variáveis contínuas. Para avaliação da normalidade das variáveis contínuas foi realizado o teste de Kolmogorov-Smirnov, considerando $p>0,05$ como distribuição normal. $O$ teste qui-quadrado de Pearson, exato de Fischer e a razão de Verossimilhança foram usados para comparar as proporções das variáveis categóricas. Os dados dos campos não preenchidos das notificações foram considerados como "sem identificação" e não foram expressos nos resultados das tabelas.

Para a tabulação dos dados, utilizou-se o programa Microsoft Excel® 2010 e para o processamento e a análise dos dados utilizou-se o programa SPSS (do inglês, Stastistical Package for the Social Sciences) versão 21.0.

\subsection{Considerações éticas}

As questões éticas relacionadas às pesquisas com seres humanos foram atendidas conforme previsto na Resolução 466/12 do Conselho Nacional de Saúde/Ministério da Saúde (BRASIL, 2012) mediante aprovação pelo Comitê de Ética em Pesquisa da Universidade Estadual do Sudoeste da Bahia (CEP-UESB), sob o parecer de nº 3.050.237. 


\section{Resultados}

Foram contabilizadas 151 notificações de erros de medicação durante o período de estudo. Destas, 78 (51,7\%) de prescrição, 69 (45,7\%) foram de erros de administração e 4 (2,6\%) de dispensação. A frequência de notificação de erros por sexo foi de $78(51,7 \%)$ para o feminino e $73(48,3 \%)$ para o masculino. Pacientes com idade $>60$ anos foram mais acometidos pelos erros de medicação, 87 (58\%) deste total. A maior frequência de notificações por setor se deu nas UTI e considerando o tipo de erro, 39 (50\%) foram erros de prescrição, 2 (50\%) de dispensação e 29 (42\%) de administração, totalizando $70(46,4 \%)$ erros de medicação (Tabela 1$)$.

Foi verificado associação significativa entre os tipos de erros de administração e a variável setor de internamento $(\mathrm{p}<0,001)$, sendo a UTI mais frequente.

Tabela 1 - Associação entre variáveis sociodemográficas e clínicas dos pacientes envolvidos em notificações de erros de medicação e o tipo de erro em um hospital geral de urgência e emergência. Bahia, Brasil, 2019.

\begin{tabular}{|c|c|c|c|c|c|}
\hline \multirow{2}{*}{ Variáveis } & \multicolumn{4}{|c|}{ Tipos de erro $N(\%)$} & \multirow{2}{*}{ p-valor* } \\
\hline & Prescrição & Dispensação & Administração & Total & \\
\hline \multicolumn{6}{|l|}{ Sexo } \\
\hline Feminino & $44(56,4)$ & $3(75,0)$ & $31(44,9)$ & $78(51,7)$ & \multirow{2}{*}{0,243} \\
\hline Masculino & $34(43,6)$ & $1(25,0)$ & $38(55,1)$ & $73(48,3)$ & \\
\hline \multicolumn{6}{|l|}{ Idade } \\
\hline $0-59$ anos & $31(39,7)$ & $2(50,0)$ & $30(44,1)$ & $63(42,0)$ & \multirow{2}{*}{0,821} \\
\hline$\geq 60$ anos & $47(60,3)$ & $2(50,0)$ & $38(55,9)$ & $87(58,0)$ & \\
\hline \multicolumn{6}{|l|}{ Setor } \\
\hline UTI & $39(50,0)$ & $2(50,0)$ & $29(42,0)$ & $70(46,4)$ & \multirow{3}{*}{0,001} \\
\hline Emergência & $31(39,7)$ & $0(0)$ & $14(20,3)$ & $45(29,8)$ & \\
\hline Clínicas & $8(10,3)$ & $2(50,0)$ & $26(37,7)$ & $36(23,8)$ & \\
\hline
\end{tabular}

Fonte: Elaboração dos autores. UTI: unidade de terapia intensiva. Clínicas: médica, neurológica, cirúrgica, vascular, pediatria e internamento não especificado. *Quiquadrado de Pearson e Exato de Fischer considerando $\mathrm{p}<0,05$.

Os erros de medicação, incluindo administração, dispensação e prescrição, foram distribuídos por setor conforme apresentado na Tabela 2. Foram apresentados 12 erros de medicação, sendo que os 3 mais frequentes foram: dose incorreta 64 $(42,4 \%)$, omissão do medicamento $40(26,5 \%)$ e horário de administração12 $(7,9 \%)$. O setor de atendimento em que foi evidenciado um maior quantitativo de notificações de erro foi nas UTI com 70 (46,3\%), seguidas das salas da emergência com $45(29,8 \%)$ e outras clínicas com $36(23,8 \%)$.

Os erros de medicação ocorrem em frequências diferentes $(p<0,001)$ em cada setor- Sendo que na UTI, as notificações apontam como maior erro a dose incorreta $(45,7 \%)$, comparado com o setor clínicas $(22,2 \%)$. A segunda maior frequência de erros (omissão do medicamento) foi apontada no setor clínicas com $75 \%$ das notificações, enquanto que no setor emergência foi de $11,1 \%$, conforme a Tabela 2. 
Tabela 2 - Frequência de erros de medicação por setores de internamento em um hospital geral de urgência e emergência. Bahia, Brasil, 2019.

\begin{tabular}{|c|c|c|c|c|}
\hline Erro & $\begin{array}{c}\text { UTI } \\
\text { N (\%) }\end{array}$ & $\begin{array}{c}\text { Emergência } \\
\mathbf{N}(\%)\end{array}$ & $\begin{array}{c}\text { Clínicas } \\
\mathbf{N}(\%)\end{array}$ & p-valor* \\
\hline Dose incorreta & $32(45,7)^{\mathrm{a}, \mathrm{b}}$ & $24(53,3)^{\mathrm{a}}$ & $8(22,2)^{b}$ & \multirow{12}{*}{$<0,001$} \\
\hline Omissão do medicamento & $8(11,4)^{\mathrm{a}}$ & $5(11,1)^{\mathrm{a}}$ & $27(75)^{b}$ & \\
\hline Horário da administração & $10(14,3)^{\mathrm{a}}$ & $2(4,4)^{\mathrm{a}}$ & $0(0)^{\mathrm{a}}$ & \\
\hline Via de administração & $6(8,6)^{\mathrm{a}}$ & $2(4,4)^{\mathrm{a}}$ & $0(0)^{\mathrm{a}}$ & \\
\hline Técnica da administração & $3(4,3)^{\mathrm{a}}$ & $5(11,1)^{\mathrm{a}}$ & $0(0)^{\mathrm{a}}$ & \\
\hline Medicamento errado & $3(4,3)^{\mathrm{a}}$ & $1(2,2)^{\mathrm{a}}$ & $1(2,8)^{\mathrm{a}}$ & \\
\hline Concentração incorreta & $2(2,9)^{\mathrm{a}}$ & $0(0)^{\mathrm{a}}$ & $0(0)^{\mathrm{a}}$ & \\
\hline Medicamento contraindicado & $2(2,9)^{\mathrm{a}}$ & $1(2,2)^{\mathrm{a}}$ & $0(0)^{\mathrm{a}}$ & \\
\hline Frequência da administração & $1(1,4)^{\mathrm{a}}$ & $4(8,9)^{\mathrm{a}}$ & $0(0)^{\mathrm{a}}$ & \\
\hline Duplicidade terapêutica não justificada & $1(1,4)^{\mathrm{a}}$ & $1(2,2)^{\mathrm{a}}$ & $0(0)^{\mathrm{a}}$ & \\
\hline Incompatibilidade & $1(1,4)^{\mathrm{a}}$ & $0(0)^{\mathrm{a}}$ & $0(0)^{\mathrm{a}}$ & \\
\hline Preparação & $1(1,4)^{\mathrm{a}}$ & $0(0)^{\mathrm{a}}$ & $0(0)^{\mathrm{a}}$ & \\
\hline Total & $70(100)$ & $45(100)$ & $36(100)$ & \\
\hline
\end{tabular}

Fonte: elaboração dos autores. UTI: Unidades de Terapia Intensiva Clínicas: médica, neurológica, cirúrgica, vascular, pediatria e internamento não especificado. Emergência: sala verde, sala amarela, sala de especialidades e sala vermelha. *Qui-quadrado de Pearson, com teste de Bonferroni, considerando $\mathrm{p}<0,05$.

Os medicamentos foram classificados conforme a ATC, nível 1, resultando em 10 categorias. As mesmas foram ordenadas de forma crescente, destacando-se as 3 mais envolvidas com os erros notificados, que foram: medicamentos do trato alimentar e metabolismo com 39 (25,8\%), seguido de sistema cardiovascular com 36 (23,8\%) e anti-infecciosos de uso sistêmico com $26(17,2 \%)$, as demais classes somaram um total de $50(33,2 \%)$, conforme a Tabela 3. 
Tabela 3 - Tipos de erros associados com a classificação ATC, nível 1 em um hospital geral de urgência e emergência. Bahia, Brasil, 2019.

\begin{tabular}{lccc}
\hline \multicolumn{1}{c}{ Classe ATC } & \multicolumn{3}{c}{ Tipos de erros N (\%) } \\
\cline { 2 - 4 } & Administração & Dispensação & Prescrição \\
\hline Trato alimentar e metabolismo & $12(17,9)$ & $0(0)$ & $27(36,5)$ \\
Sistema cardiovascular & $13(19,4)$ & $0(0)$ & $23(31,1)$ \\
Anti-infecciosos de uso sistêmico & $18(26,9)$ & $2(50)$ & $6(8,6)$ \\
Sistema nervoso & $5(7,5)$ & $2(50)$ & $(10,8)$ \\
Preparações hormonais sistêmicas, excluindo & $8(11,9)$ & $0(0)$ \\
hormônios sexuais e insulinas & $3(4,5)$ & $0(0)$ & $4(4,8)$ \\
Sistema respiratório & $2(3)$ & $0(0)$ & $4(5,4)$ \\
Órgãos sensoriais & $4(6)$ & $0(0)$ & $1(1,4)$ \\
Sangue e Órgãos de formação de sangue & $1(1,5)$ & $0(0)$ & $1,4)$ \\
Sistema cardiovascular/trato alimentar e & $1(1,5)$ & $0(0)$ & $0(0)$ \\
metabolismo & $67(46,2)$ & $4(2,8)$ & $74(51)$ \\
Antiparasitários, inseticidas e repelentes & & & \\
\hline Total & & & \\
\hline
\end{tabular}

Fonte: Elaboração dos autores. ATC: Anatomic Terapeutic Chemical.

Quanto aos profissionais que realizaram as notificações, obteve-se um total de $116(76,8 \%)$ notificadas por farmacêuticos, seguido de 20 (13,2\%) por técnicos de enfermagem, 4 (2,6\%) por enfermeiros e em 11 (7,3\%) não havia identificação do profissional notificador, Tabela 4. Foi verificado associação significativa entre os tipos de erros de administração e a variável categoria profissional $(\mathrm{p}<0,001)$.

Tabela 4 - Frequência de notificações por categoria profissional em um hospital geral de urgência e emergência. Bahia, Brasil, 2019.

\begin{tabular}{cccc}
\hline Categoria Profissional & Fa & Fr (\%) & p-valor* \\
\hline Farmacêutico & 116 & 76,8 & $<0,001$ \\
Técnicos de enfermagem & 20 & 13,2 & \\
Enfermeiro & 4 & 2,6 & \\
\hline Total & 151 & 100 & \\
\hline
\end{tabular}

Fonte: Elaboração dos autores. Fa: frequência absoluta. Fr: frequência relativa. *Qui-quadrado de Pearson, considerando p<0,05.

\section{Discussão}

Os principais achados deste estudo evidenciaram uma alta frequência de erros de medicação, notificados em um hospital público, principalmente os erros de prescrição e administração, fato este que destaca a necessidade de reflexão sobre as práticas de segurança que têm sido adotadas neste ambiente, no que que compete à gestão da farmacoterapia. Por outro lado, essas evidências apontam também para a importância de se fomentar mais investigações sobre os fatores relacionados a estes eventos adversos, posto que são evitáveis, no sentido de sensibilizar os profissionais a aderirem a cultura de segurança, bem como a cultura da notificação dos erros de medicação em face de suas ocorrências. 
Observou-se que a frequência de notificação de erros foi maior para faixa etária acima de 60 anos, o que corrobora com achados de outros estudos em que os eventos adversos relacionados a medicamentos vêm sendo citado como os incidentes de segurança mais frequentes na população idosa (Maher, Hanlon \& Hajjar, 2014; Newbould et al., 2017), sendo que as principais causas apontadas são: idade avançada, múltiplas doenças e polifarmácia (Valle, Cruz, \& Santos, 2017).

Dentre os setores, destacou-se maior frequência de notificações de erros de medicação nas UTI, onde a vigilância e suporte ao paciente são maiores. Porém outro estudo aponta que a complexidade também exerce influência, o que os deixam mais vulneráveis a ocorrência de eventos adversos (Karimian et al., 2018).

Neste cenário, existem vários fatores que contribuem para erros de medicação nas UTI, destacando-se a colaboração interprofissional inadequada, falta de atenção aos princípios de segurança de medicamentos, conhecimento inadequado sobre medicamentos, determinantes ambientais, falta de farmacêutico clínico no setor, entre outros (Farzi, Farzi, \& limohammadi, 2016; Farzi et al., 2018; Karimian et al., 2018).

$\mathrm{Na}$ Espanha foram realizados alguns estudos que apontaram que existem $22 \%$ de risco de um paciente internado em uma UTI ser acometido por erros de medicação, sendo que a probabilidade maior que este aconteça é durante a prescrição e administração de medicamentos (Willian, 2007), fatos que coincidem com os resultados encontrados nas notificações aqui analisadas. Em estudo realizado em UTI (Farzi et al., 2017) foi constatado que $80 \%$ dos participantes, em um mês, foram acometidos por erros, pelo menos uma vez, sendo que houve um aumento para 91,2\% no ano seguinte (Farzi et al., 2018).

Entretanto, o fato de haver mais notificações neste setor não infere que este seja o local em que mais ocorrem erros. $O$ cuidado com pacientes mais graves mobiliza os profissionais a serem mais vigilantes, o que os leva a terem ações para aumentar a garantia da segurança do paciente (Garfield et al., 2009), e consequentemente notificarem mais.

No presente estudo, as notificações do tipo de erro de prescrição foram as mais frequentes em relação às outras, tendo como maior ocorrência a dose incorreta dos medicamentos, o que coincide com um estudo nacional que analisou 230 erros de medicação e identificou que 16,5\% destes estavam relacionados à dose (Willian, 2007), sendo esses erros apontados pelos autores, como reflexos da falta de conhecimento sobre medicamentos específicos e dificuldade no manuseio de alguns equipamentos (Willian, 2007). Neste contexto, Suclupe et al. (Suclupe et al., 2020) também discute que os dados incompletos e deficiência no controle das dosagens estão relacionados aos riscos de erros de medicação na prescrição.

O segundo tipo de erro mais notificado foi o de administração, seguido do de dispensação. Dentre as notificações de erro de administração, a omissão do medicamento, por falta do mesmo no hospital, foi a mais frequente. O estudo de Silva, et al. (Silva et al., 2017) pontuou que o principal determinante dos erros de medicação foi a omissão por indisponibilidade dos medicamentos na farmácia do hospital e que existem fatores externos e internos que determinam a indisponibilidade, sendo importante o equilíbrio entre os mesmos para que seja evitada a escassez dos medicamentos.

Ademais, a segurança no processo de atendimento é comprometida pela falta de medicamento que é gerada por falhas no processo de logística, o que leva uma maior probabilidade de ocorrência de eventos. Sendo assim destaca-se a importância da incorporação de uma logística moderna com ferramentas de gestão eficientes que propiciem um planejamento adequado evitando erros de omissão por desabastecimentos de insumos (Willian, 2007).

Neste estudo as notificações de erros de dispensação tiveram a menor ocorrência. Um estudo multicêntrico, realizado em quatro hospitais brasileiros nas cidades de Recife, Ribeirão Preto, Goiânia e São Paulo, mostrou que esses tipos de erros aconteceram em uma taxa de 1 a $24 \%$, sendo que a separação do produto errado foi um dos motivos e ocorre principalmente por terem aparência ou nomes semelhantes (Miasso et al., 2006). Outro estudo realizado em hospitais do Iran, mostrou que, de 61 erros, 4 foram relacionados à dispensação, tendo como justificativa o fato do hospital estudado possuir um sistema de distribuição informatizado com identificação por código de barras, o que contribui para prevenção da ocorrência de erros, mas não os impede, podendo justificar a baixa ocorrência em relação aos hospitais que não possuem sistema de distribuição 
informatizado (Silva, Faveri, \& Lorenzini, 2014). Fato este que coincide com a realidade do hospital estudado.

No que se refere a classificação dos grupos terapêuticos, segundo ATC nível 1, no presente estudo, as classes que estiveram envolvidas com maior nos erros foram os medicamentos destinados ao trato alimentar e metabolismo, sistema cardiovascular e anti-infecciosos de uso sistêmico, por serem as classes de medicamentos mais prescritas em hospitais. Os dados encontrados em outro estudo, realizado em UTI de hospital de ensino no Irã também apontam os anti-infecciosos sistêmicos e trato alimentar e metabolismo estão dentre os grupos terapêuticos de maior frequência entre erros de medicação (Irajpour, Farzi, Saghaei, \& Ravaghi, 2019). Porém, em outro estudo realizado em no estado de Goiás, mais da metade dos erros de prescrição identificados envolve medicamentos que atuam no sistema nervoso e no sistema cardiovascular (Silva et al 2011).

Cabe destacar a importância de se discutir práticas seguras, direcionadas especificamente ao uso de anti-infecciosos de uso sistêmico, dado o risco de desenvolvimento da resistência microbiana, que além de proporcionar maiores problemas clínicos aos pacientes, são responsáveis também por elevação dos custos e aumento do tempo de internamento em leitos hospitalares (Loureiro et al., 2016).

Quanto aos profissionais, os farmacêuticos foram os responsáveis pela maioria das notificações. Esse fato pode ser atribuído ao desenvolvimento recente de algumas ações de farmacovigilância e farmácia clínica realizada por farmacêuticos Residentes, o que anteriormente não acontecia. Porém estudo com avaliações de relatórios do Food and Drug Administration (FDA) aponta os enfermeiros como os responsáveis pela maior parte das notificações de eventos relacionados aos medicamentos (Karimian et al., 2018).

Dentre as formas de detecção de incidentes, a notificação voluntária é o método mais utilizado. Entretanto, possui limitações como a falta de tempo para fazer a notificação, receio pelos profissionais em causar confrontos entre os membros das equipes, resistência em relatar os próprios erros, sistemas de informação inadequados, falta de conhecimento sobre a importância de notificar, falta de ações resolutivas resultantes dos problemas evidenciados nas notificações, o que resulta na constatação de subnotificação (Garrouste-Orgeas et al., 2012). Este fato é apontado em estudos nacionais e internacionais e a subnotificação ou não notificação se dá devido ao receio e medo diante da ocorrência dos erros (Alves \& Albuquerque, 2019; Farzi et al., 2017). No entanto estudo realizado em hospital federal no estado do Rio de Janeiro apresenta uma aumento na adesão a realização de notificações com a melhora da cultura de segurança no trabalho (Ribeiro \& Novaes, 2021).

Uma revisão que discutiu sobre os motivos para a não notificação de incidentes de segurança do paciente por profissionais no contexto dos serviços de saúde brasileiros evidenciou que vários autores recomendam que a cultura punitiva deva ser vencida, além do investimento em capacitação e sensibilização dos profissionais, incentivo as notificações e implementação de ações para reduzir a ocorrência de eventos adversos (Alves, Carvalho, \& Albuquerque, 2019).

Como estratégia para melhorar a segurança dos pacientes e consequentemente diminuir os erros, estudos apontam para programas de educação continuada sobre medicação (Farzi et al., 2017; Farzi et al., 2018; Karimian et al., 2018). Brock et al. (Brock et al., 2013) mostraram que, após a implementação de um programa de educação interprofissional de segurança de medicamentos, houve uma redução significativa nos relatos e taxas de erros de medicação. Estudo, realizado em hospital universitário no oeste do Paraná, mostra que intervenções farmacêuticas e conferência das prescrições antes da dispensação reduzem erros de medicação (Ferreira, Pereira, 2021). A realização de educação interprofissional é importante e proporciona um atendimento mais abrangente, além de melhorar a relação entre a equipe de saúde e algumas competências necessárias como uma comunicação eficaz, escuta ativa, respeito, pontualidade, entre outras (Garfield et al., 2009). Sendo assim, sugere-se enfoque na realização de ações já pautadas como eficientes frente aos resultados encontrados.

Diante destes resultados, pontuamos que alguns fatores, como o período de tempo do estudo, a ausência de algumas informações nas fichas de notificação e a possível subnotificação, foram considerados como limitações deste estudo. 
Entretanto, esses achados trazem informações aos gestores de risco para que possam direcionar ações preventivas na ocorrência de erros de medicação, promoção de educação continuada, esclarecimentos e incentivo para notificação e consequentemente uma melhoria na assistência à saúde e segurança dos pacientes.

\section{Conclusão}

Neste estudo constatou-se uma maior frequência de notificações dos tipos de erros de prescrição e administração do que de dispensação. Os erros mais frequentes encontrados foram os de dose e omissão do medicamento. As UTI foram os setores que mais notificaram a ocorrência de erros de medicação, seguida das salas da emergência e demais clínicas.

Diante da frequência de notificações dos erros identificados e considerando que erros de medicação são eventos evitáveis, sugere-se que esforços sejam dedicados na busca de mais informações para uma melhor avaliação e conhecimento das consequências e possíveis danos relacionados aos erros de medicação, assim como para o planejamento e desenvolvimento de ações preventivas e educativas para promoção de uma cultura de segurança no uso dos medicamentos, esperando que haja redução desses eventos, e promovendo assim uma assistência à saúde de maior qualidade e segurança para o paciente. Sendo assim, temas que abordem qual a metodologia de notificação das instituições, danos causados por erros de medicação, causas que levam a ocorrências de erros e ações corretivas baseada nas análises desses dados, podem ser sugeridos para futuros estudos.

\section{Referências}

Alves, M. de F. T., Carvalho, D. S. de, \& Albuquerque, G. S. C. de. (2019). Motivos para a não notificação de incidentes de segurança do paciente por profissionais de saúde: revisão integrativa. Ciência \& Saúde Coletiva, 24(8), 2895-2908. https://dx.doi.org/10.1590/1413-81232018248.23912017

Anacleto, T. A., Rosa, M. B., Miranda, H. M., \& Martins, M. A. P. (2010). Erros de medicação. Site Conselho Federal de Farmácia. http://www.cff.org.br/sistemas/geral/revista/pdf/124/encarte_farmaciahospitalar.pdf

Brasil. Agência Nacional de Vigilância Sanitária. Bulário Eletrônico. Disponível em: https://consultas.anvisa.gov.br/\#/bulario/

Brasil. Ministério da Saúde. Resolução 466/12. O Plenário do Conselho Nacional de Saúde resolve aprovar diretrizes e normas regulamentadoras de pesquisas envolvendo seres humanos. 2012.

Brock, D., Abu-Rish, E., Chiu, C., Hammer, D., Wilson, S.; Vorvick, L., Blondon, K., Schaad, D., Liner, D. \& Zierler, B. (2013). Interprofessional education in team communication: working together to improve patient safety. BMJ Qual Saf., 22, 414-23

CFF. Conselho Federal de Farmácia. (2018, January 16). Informações sobre medicamentos, apenas de fontes seguras. Conselho Federal de Farmácia Site. https://www.cff.org.br/noticia.php?id=4786

Farzi, S., Farzi, S., \& Alimohammadi, N. (2016). Medication errors by the Intensive care units'nurses and the preventive strategies. JAP, 33-45. http://jap.iums.ac.ir/article-1-5231-en.html

Farzi, S., Irajpour, A., Saghaei, M., \& Ravaghi, H. (2017). Causes of Medication Errors in Intensive Care Units from the Perspective of Healthcare Professionals. Journal of research in pharmacy practice, 6(3), 158-165. https://doi.org/10.4103/jrpp.JRPP_17_47

Farzi, S., Saghaei, M., Irajpour, A., \& Ravaghi, H. (2018). The most frequent and important events that threaten patient safety in intensive care units from the perspective of health-care professionals'. Journal of research in medical sciences: the official journal of Isfahan University of Medical Sciences, 23 , 104. https://doi.org/10.4103/jrms.JRMS_140_18

Ferreira, F. S., Pereira, T. A., Souza, B. P., \& Sanches, A. C. C. (2021) The pharmacist's role in preventing medication errors. Research, Society and Development, [S. l.], 10(3), e18310313280, 2021. DOI: 10.33448/rsd-v10i3.13280.

Garfield, S., Barber, N., Walley, P., Willson, A., \& Eliasson, L. (2009). Quality of medication use in primary care--mapping the problem, working to a solution: a systematic review of the literature. BMC Med. Published. https://doi.org/10.1186/1741-7015-7-50

Garrouste-Orgeas, M., PHILIPPART, F., Bruel, C., Max, A., Lau, N., \& Misset, B. (2012). Overview of medical errors and adverse events. Ann Intensive Care. Published. https://doi.org/10.1186/2110-5820-2-2

Irajpour, A., Farzi, S., Saghaei, M, \& Ravaghi, H. (2019) Effect of interprofessional education of medication safety program on the medication error of physicians and nurses in the intensive care units. J Edu Health Promot, 8, 196.

Karimian, Z., Kheirandish, M., Javidnikou, N., Asghari, G., Ahmadizar, F., Dinarvand, R. (2018). Medication Errors Associated with Adverse Drug Reactions in Iran (2015-2017): A P-Method Approach. International Journal of Health Policy and Management, 7(12), 1090-1096. doi: 10.15171/ijhpm.2018.91 
Loureiro, R. J., Roque, F., Rodrigues, A. T., Herdeiro, M. T., \& Ramalheira, E. (2016). O uso de antibióticos e as resistências bacterianas: breves notas sobre a sua evolução. Revista Portuguesa de Saúde Pública, 34, 77-87. https://doi.org/10.1016/j.rpsp.2015.11.003

Maher, R. L., Hanlon, J., \& Hajjar, E. R. (2014). Clinical consequences of polypharmacy in elderly. Expert Opin Drug Saf, 13, 57-65. https://doi.org/10.1517/14740338.2013.827660

Meira, G. M.; Souza, T. S.; Lemos, L. B. \& Lemos, G. S. (2020). Prescription and administration errors of antimicrobials in a pediatric inpatient unit. Rev Bras Farm Hosp Serv Saude, 11(4):0502.

Mendes, J. R.; Lopes, M. C. B. T.; Vancini-Campanharo, C. R.; Okuno, M. F. P. \& Batista, R. E. A. (2018). Tipos e frequência de erros no preparo e na administração de medicamentos endovenosos. Einstein (São Paulo) [online]. 16 (3), eAO4146. http://dx.doi.org/10.1590/s1679-45082018ao4146.

Miasso, A. I., Grou, C. R., Cassiani, S. H. B., Silva, Ana Elisa Bauer de Camargo, \& Fakih, Flávio Trevisan. (2006). Erros de medicação: tipos, fatores causais e providências tomadas em quatro hospitais brasileiros. Revista da Escola de Enfermagem da USP, 40(4), 524-532. https://dx.doi.org/10.1590/S008062342006000400011

National Coordinating Council for Medication Error Reporting and Prevention. (NCCMERP). What is a medication error? Nova York: National Coordinating Council for Medication Error Reporting and Prevention; 2015. http://www.nccmerp.org/aboutmedication-errors

Néri, E. D. R., Gadêlha, P. G. C., Maia, S. G., Pereira, A. G. S.; Almeida, P. C., Rodrigues, C. R. M., Portela, P. M. \& Fonteles, M. M. F. (2011). Erros de prescrição de medicamentos em um hospital brasileiro. Rev Assoc Med Bras, 57, 306-314.

Newbould, V., Le Meur, S., Goedecke, T., \& Kurz, X. (2017). Medication errors: a characterization of spontaneously reported cases in eudravigilance. Drug Saf, 40, 1241-1248. https://doi.org/10.1007/s40264-017-0569-3

OMS. Organização Mundial de Saúde. (2019). Índice químico terapêutico anatômico (ATC) com doses diárias definidas (DDDs). WHO Collaborating Centre for Drug Statistics Methodology Site. http://www.whocc.no/atcddd

OMS. Organização Mundial de Saúde. Medication without harm: WHO’s third global patient safety challenge. Geneva: WHO, [2017]. <http://www.who.int/patientsafety/medication-safety/en/>.

Pereira, A. S., Shitsuka, D. M., Parreira, F. J., \& Shitsuka, R. (2018). Metodologia da pesquisa científica (1 ${ }^{\text {a }}$ ed.): Universidade Federal de Santa Maria, Núcleo de Tecnologia Educacional. https://repositorio.ufsm.br/bitstream/handle/1/15824/Lic_Computacao_Metodologia-Pesquisa-Cientifica.pdf?sequence=1>.

Ribeiro, A. E. C. \& Novaes, C. de O. (2019) Characterization of incidents in a Federal Public Hospital between the years 2014 and 2019. Research, Society and Development, [S. l.], 10(4), e30510414080, 2021. DOI: 10.33448/rsd-v10i4.14080.

Santi, T., Beck, C. L. C., da Silva, R. M.; Zeitoune, R. G., Tonel, J. Z. \& do Reis, D. A. Martins. (2014). Erro de medicação em um hospital universitário: percepção e fatores relacionados. Enfermería Global, 13, 160-183.

Secretaria Estadual de Saúde da Bahia (SESAB). Regiões de Saúde do Estado da Bahia: Municípios da Região de Saúde Jequié. 2018. <http://www1.saude.ba.gov.br/mapa_bahia/result_REGIAO_SAUDEch.asp?REGIAO_SAUDE=Jequi\%E9>.

Silva, A. E. B. C., Reis, A. M. M., Miasso, A. I., Santos, J. O., \& Cassiani, S. H. B. (2011). Adverse drug events in a sentinel hospital in the State of Goiás, Brazil. Revista Latino-Americana de Enfermagem, 19(2), 378-386. https://dx.doi.org/10.1590/S0104-11692011000200021

Silva, E. F., de Faveri, F., \& Lorenzini, L. (2014). Errores de medicación en el ejercicio de la enfermería: una revisión integrativa. Enfermería Global, 13(34), 330-337. Recuperado en 19 de mayo de 2021, de http://scielo.isciii.es/scielo.php?script=sci_arttext\&pid=S169561412014000200016\&lng=es\&tlng=es

Silva, J. S. D. S.; Almeida, P. H. R. F.; Perini, E.; Pádua, C. A. M.; Rosa, M. B. \& Lemos, G. S. (2017). Erros de prescrição e administração envolvendo um medicamento potencialmente perigoso. Rev Enferm UFPE on Line, 11, 3707-3717.

Suculupe, S., Martinez-Zapata, M.J., Mancebo, J., Font-Vaquer, Assumpta., Castillo-Masa, A.M., Viñolas, I., Morán, I. \& Robleda, Gemma. (2020). Medication errors in prescription and administration in critically ill patients. Journal of Advanced Nursing. Published. https://doi.org/10.1111/jan.14322

Valle, M. M. F., Cruz, E. D. A. \& Santos, T. (2017). Incidentes com medicamentos em unidade de urgência e emergência: análise documental. Revista Da Escola de Enfermagem Da USP, 51. https://doi.org/10.1590/s1980-220x2016033303271

Watanabe, J. H., Mcinnis, T. \& Hirsch, J. D. (2018). Cost of Prescription Drug-Related. Morbidity and Mortality. Annals of Pharmacotherapy, 1-9.

Williams, D. J. P. (2007). Medication errors. Journal-Royal College of Physicians of Edinburgh, 37. 